\title{
Primary optic nerve sheath meningioma
}

\author{
J E WRIGHT, A A McNAB, AND W I McDONALD \\ From the Orbital Clinic, Moorfields Eye Hospital, City Road, London EC1V 2PD
}

\begin{abstract}
SUMMARY Fifty patients with optic nerve sheath meningiomas have been reviewed with a followup of up to 15 years. The median age at onset of their symptoms was $40 \cdot 0$ years. The majority were middle aged females with a slowly progressive lesion. More aggressive lesions were encountered in a younger, predominantly male group of patients with frequent intracranial involvement. Our experience indicates that a more aggressive surgical approach to these lesions is needed to prevent this sequence of events. Meningiomas in older individuals often do not need treatment, though radiotherapy can be beneficial.
\end{abstract}

Meningiomas within the orbit may arise from the meninges of the intraorbital optic nerve (primary optic nerve sheath meningioma) or from the meninges of the optic canal or sphenoid bone. Very rarely orbital meningiomas appear to arise from ectopic arachnoid cells separate from any meningeal tissue. This paper deals with meningiomas primary to the optic nerve sheath. In the past we treated these by local excision. In the older patients there have been few recurrences, but it has become increasingly apparent that the younger the patient the more aggressive the growth and spread of the meningioma. Our management of these patients has therefore changed. This paper details our experience and our suggested treatment in patients of differing age.

\section{Patients and methods}

The diagnosis of optic nerve sheath meningioma has been made in 50 patients from a total of over 3000 seen in the Orbital Clinic at Moorfields Eye Hospital. Histological confirmation has been obtained in 44 cases. The remaining six have not undergone surgery, and the diagnosis has been made on the basis of typical clinical and radiological features. The presence of good vision has been the major reason for not operating on these patients, but in a few patients old age and general medical problems have influenced the decision.

In many patients it was not clear at the time of presentation, even with sophisticated methods of investigation, whether the meningioma had arisen Correspondence to J E Wright, FRCS, Moorfields Eye Hospital, City Road, London EC1V 2PD. within the orbital optic nerve sheaths (primary optic nerve sheath meningioma) or from the meninges of the optic canal or adjacent sphenoid bone. However, we have included only patients who clinically presented as if their meningioma was primarily affecting the optic nerve sheath and excluded those who quite clearly had meningiomas of the sphenoid which secondarily affected the optic nerve. In the group reported on here it is quite probable that some of the meningiomas were not primary to the optic nerve sheaths in the strictest sense, but such a distinction is, we believe, both unhelpful and unnecessary, as the decisions to be made about the patient's management are essentially the same.

In all cases a detailed clinical history was obtained and a full ocular and neurological examination performed. Routine skull films with optic canal views were always obtained. All but a few patients have undergone CT scanning, and a small number have also had angiography and magnetic resonance imaging (MRI).

Thirteen patients have been referred for craniotomy, the oldest of these being 52 at the time of surgery. Orbitotomy, usually via a lateral approach, has been performed in all other operated cases and also on many of the craniotomy patients. In those undergoing orbital surgery and attempted excision our initial approach involved stripping the nerve sheath and meningioma from the underlying nerve in the hope of preserving vision. In all these cases the eye was rendered blind as a result of the surgery and there was a high incidence of extradural orbital recurrence. In a small number an attempt to preserve vision was made by slitting open the nerve sheath to 
Table 1 Age and sex data

\begin{tabular}{|c|c|c|c|}
\hline Total patients & & 50 & \\
\hline $\begin{array}{l}\text { Sex } \\
\text { Involved optic nerve }\end{array}$ & $\begin{array}{l}\text { Male } 17 \\
\text { Right } 20\end{array}$ & & $\begin{array}{l}\text { Female } 33 \\
\text { Left } 30\end{array}$ \\
\hline $\begin{array}{l}\text { Age at onset } \\
\text { All patients } \\
\text { Males } \\
\text { Females }\end{array}$ & $\begin{array}{l}\text { Mean } \\
38 \cdot 9 \\
33 \cdot 3 \\
43 \cdot 2\end{array}$ & $\begin{array}{l}\text { Median } \\
40 \cdot 0 \\
30 \cdot 0 \\
42 \cdot 0\end{array}$ & $\begin{array}{l}\text { Range } \\
5-65 \\
5-61 \\
12-65\end{array}$ \\
\hline $\begin{array}{l}\text { Age at presentation } \\
\text { All patients } \\
\text { Males } \\
\text { Females }\end{array}$ & $\begin{array}{l}43 \cdot 2 \\
38 \cdot 5 \\
47 \cdot 2\end{array}$ & $\begin{array}{l}40 \cdot 0 \\
31 \cdot 0 \\
46 \cdot 0\end{array}$ & $\begin{array}{l}10-70 \\
10-78 \\
20-66\end{array}$ \\
\hline
\end{tabular}

decompress the nerve. In these patients meningioma recurred in the orbital fat and extraocular muscles. We now aim to remove the nerve and its sheath en bloc, whether this be via a craniotomy or an orbitotomy or a combination of the two.

\section{Results}

The age and sex distributions of the patients are presented in Table 1 and the age at onset of symptoms is shown graphically in Fig. 1. The males were clustered in the younger age group, so that of those whose symptoms began before the age of 20 years five out of six were male. Of all 13 patients aged 30 and under 10 were male, and of those 27 patients aged 40 or less 12 were male. This compares with the overall sex ratio of 17 males to 33 females. This increased number of males was statistically significant for the groups aged 20 or less and 30 or less $\left(\chi^{2}\right.$ test with Yates's correction gives $p<0.025$ and $p<0.001$ respectively), but not for those aged 40 or less $(\mathrm{p}>0 \cdot 1)$.

The presenting symptoms and their duration are detailed in Table 2. Most patients noticed loss of vision as their first symptom, and this often preceded the recognition of any proptosis by a year or more. Of

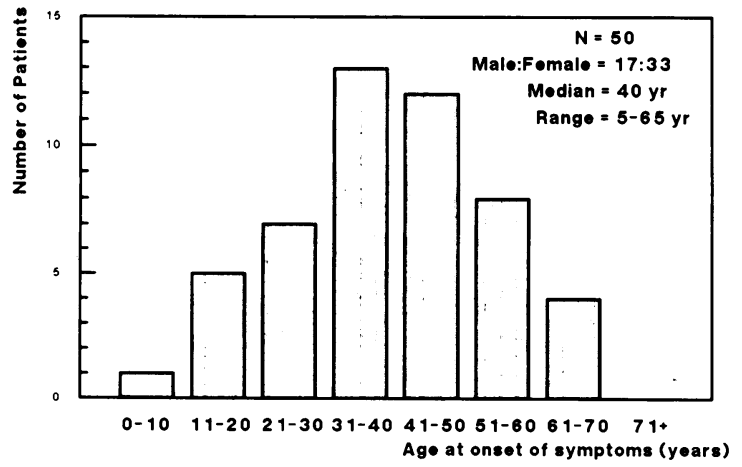

Fig. 1 Age at onset of symptoms for 50 patients with optic nerve sheath meningiomas.
Table 2 Presenting symptoms

\begin{tabular}{lrlll}
\hline Symptom & Number & \multicolumn{3}{l}{ Duration (years) } \\
\cline { 3 - 5 } & & Mean & Median & Range \\
\hline Visual loss & 45 & $4 \cdot 8$ & $3 \cdot 0$ & $0 \cdot 2-28$ \\
Proptosis & 18 & $3 \cdot 3$ & $2 \cdot 0$ & $0 \cdot 1-10$ \\
Obscurations & 7 & $0 \cdot 5$ & $0 \cdot 2$ & $0 \cdot 1-1 \cdot 5$ \\
Diplopia & 3 & & & \\
Lid swelling & 3 & & & \\
Pain & 2 & & & \\
\hline
\end{tabular}

the patients with visual obscurations one had these as his only symptom. In three patients the obscurations were gaze evoked, occurring in abduction. In two they were brought on by postural changes, and in the remaining two they were spontaneous. The vascular basis of these obscurations was proved in one patient by fluorescein angiography.' On abduction of the globe the central retinal arterial flow ceased and then returned as the eye resumed the primary position.

The clinical findings at presentation are outlined in Table 3. Almost one-third of patients $(16 / 50)$ had $6 / 18$ or better vision when first seen. The amount of proptosis was generally small (median $2.0 \mathrm{~mm}$ ) and did not correlate closely with the visual acuity, though all patients with $6 / 6$ vision had $2.0 \mathrm{~mm}$ or less proptosis (whereas those with no perception of light ranged from 0 to $8 \mathrm{~mm}$ ).

The majority of patients had an atrophic optic disc, and, in about a third of these, opticociliary shunt vessels were present. The patient with a normal disc had $6 / 6$ vision. His meningioma was discovered incidentally on CT scan for a suspected acoustic neuroma; no surgery has been performed. This patient does not have neurofibromatosis. In the whole series only one patient did, and he had the central form of the condition with bilateral acoustic neuromas (Case 1 below).

Table 3 Clinical findings at presentation

\begin{tabular}{llr}
\hline Visual acuity & Number & $(\%)$ \\
\hline $6 / 6-6 / 9$ & 10 & $(20)$ \\
$6 / 12-6 / 18$ & 6 & $(12)$ \\
$6 / 24-6 / 36$ & 4 & $(8)$ \\
6/60-Count fingers & 8 & $(16)$ \\
Hand movements-light perception & 10 & $(20)$ \\
No light perception & 12 & $(24)$ \\
\hline Proptosis & Mean & $3 \cdot 3 \mathrm{~mm}$ \\
& Median & $2 \cdot 0 \mathrm{~mm}$ \\
& Range $0-11 \mathrm{~mm}$ \\
Optic disc & Atrophic & 31 \\
& Swollen & 19 \\
& Opticociliary & 12 \\
& shunts & \\
& Normal & 1 \\
& &
\end{tabular}




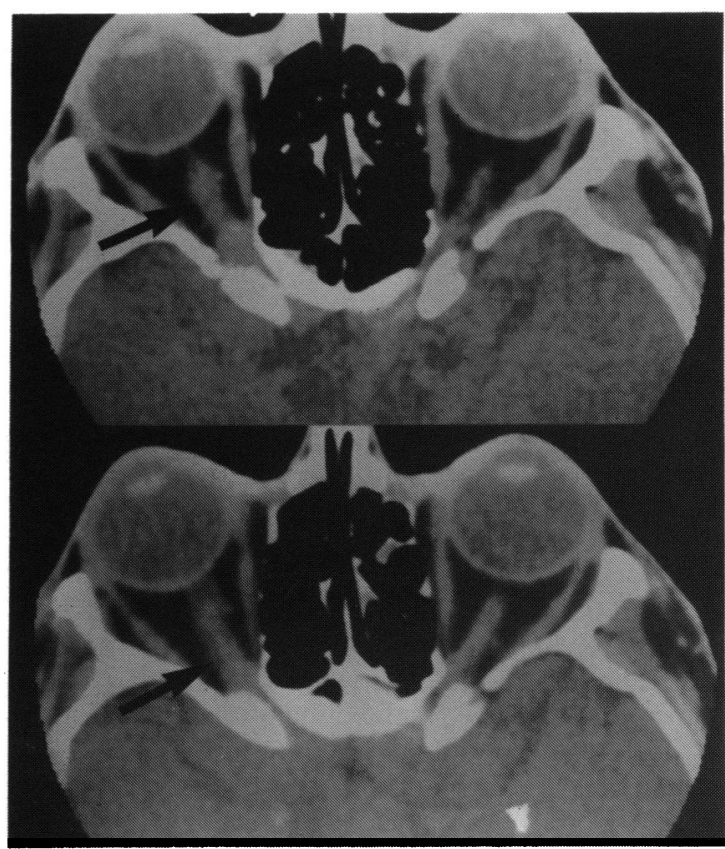

Fig. 2 Axial CT scan with contrast of a patient with diffuse enlargement of the optic nerve caused by meningioma (arrowed).

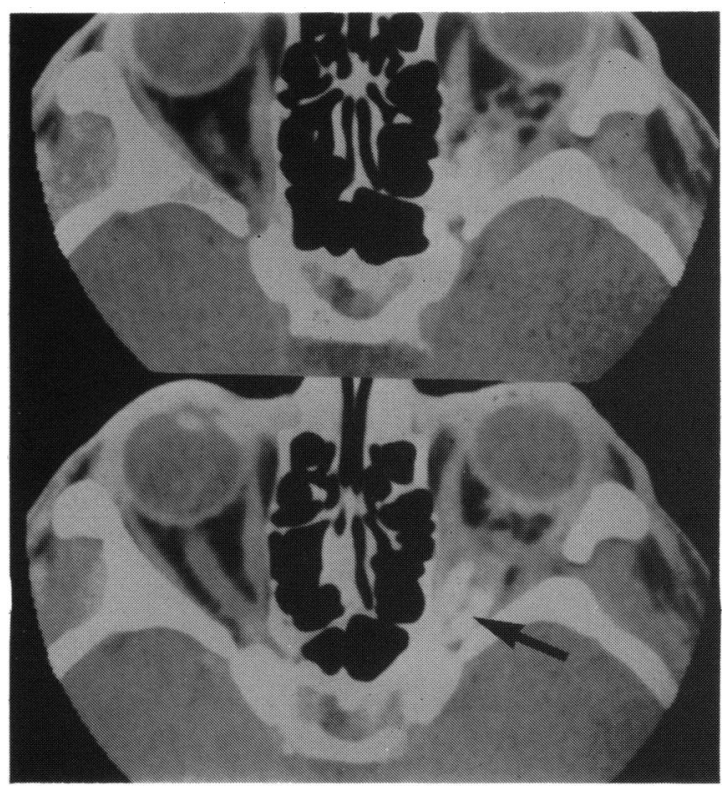

Fig. 3 Axial CT scan with contrast showing 'tramline' calcification within the sheath of the optic nerve (arrowed). This elderly patient previously had a lateral orbitotomy and excision of the anterior two-thirds of the orbital optic nerve and its sheath.

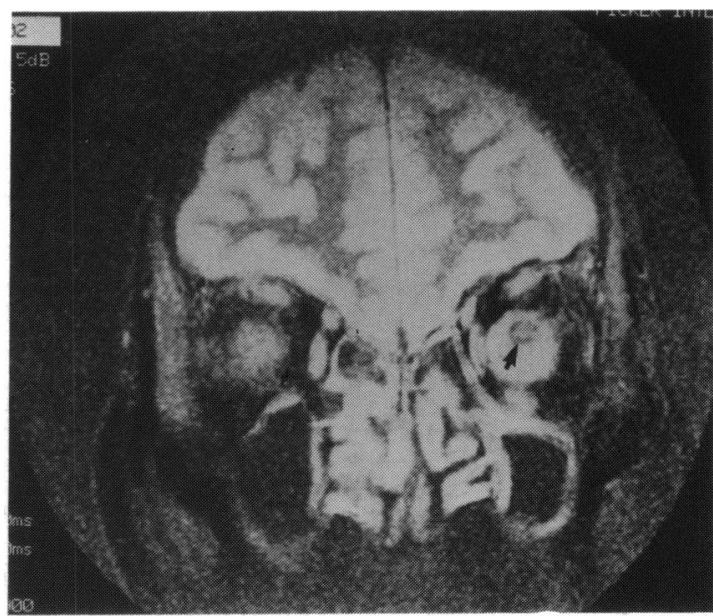

Fig. 4 Coronal MRI showing a meningioma surrounding the optic nerve (arrowed).

CT scans (Fig. 2) were performed in 47 cases. Ten patients showed definite calcification of either a 'tramline' type (Fig. 3) or scattered within the tumour on CT scan. Of the 31 cases with histopathological confirmation in whom a CT scan was performed prior to surgery and the radiologist's report was available a confident diagnosis of meningioma was made in 23. This gives a diagnostic accuracy rate of $74 \%$. Ten of these showed calcification. In four the radiologist was equivocal, reporting the presence of an optic nerve tumour only. In four the diagnosis was incorrect. Three were reported as likely to be glioma. All three of these patients were young at the time of the scan (ages 10,20, and 20 years). One patient in whom the expansion of the optic nerve was reported as meningioma was found to have cystic expansion of the optic nerve sheath. An apical meningioma not seen on the original scan was discovered only several years later. These scans were performed on equipment ranging from the prototype scanner of 1972 up to current, high resolution machines. In patients seen and scanned since 1980 the diagnostic accuracy has been over $90 \%$.

Table 4 Intracranial disease related to the age of onset of symptoms

\begin{tabular}{lll}
\hline $\begin{array}{l}\text { Age at onset } \\
\text { of symptoms }\end{array}$ & $\begin{array}{l}\text { Intracranial disease } \\
\text { detected by CT or MRI }\end{array}$ & $\begin{array}{l}\text { Total with } \\
\text { intracranial disease }\end{array}$ \\
\hline 20 years or less & $3 / 6$ & $4 / 6$ \\
$21-30$ & $2 / 7$ & $2 / 7$ \\
$31-40$ & $5 / 13$ & $7 / 13$ \\
$41-50$ & $3 / 12$ & $3 / 12$ \\
$51-60$ & $1 / 8$ & $1 / 8$ \\
$61+$ & $0 / 4$ & $0 / 4$ \\
\hline
\end{tabular}


MRI was used in only a small number of cases (Fig. 4), and its value in the assessment of optic nerve meningioma is still under review.

No patients had symptoms or signs of intracranial disease at presentation or within the course of followup. However 13 had radiological evidence of intracranial disease on CT. One further patient had intracranial disease detected only on MRI. Three patients without radiological evidence of intracranial disease were found at craniotomy to have a small amount of meningiomatous tissue emerging from the proximal end of the optic canal. One of these had had MRI in addition to CT.

There were 14 patients with radiological evidence of intracranial involvement. In addition three were found to have intracranial spread only at craniotomy, CT scans having failed to detect it. We excluded these three from the following statistical analysis, as we have tended to recommend craniotomy only for the younger patients, and this introduces a bias. Of the 14 with radiologically detected intracranial disease 10 were 40 years or less at the time of onset of their symptoms. This does not represent a statistically significant age related difference $\left(\chi^{2}\right.$ test with Yates's correction gives $\mathrm{p}<0 \cdot 25$ ). If this trend were to continue, between 2 and 3 times as many patients would be required to attain statistical significance. For patients aged 20 or less, three out of six developed intracranial disease detected with CT scan, and one other was found to have intracranial spread at craniotomy. The incidence of intracranial disease in all age groups is represented in Table 4. Five were male and 12 female, but this difference is not statistically significant. In addition there was no difference in the types of investigation performed in the different age groups, and the period of follow-up was similar in each group.

Prior surgery did not have a significant effect on the development of intracranial disease. Of the 17 patients with intracranial disease 10 had had no previous surgery. Three had a small biopsy via an orbitotomy 2,7 , and 8 years before; three had the orbital optic nerve and sheath excised via a lateral orbitotomy 7,8 , and 9 years before, and one patient had the sheath stripped from the nerve 12 years before detection of the intracranial disease.

No patient has died from meningioma in the period of follow-up, which has ranged from three months to 15 years (mean 6.8 and median 6.0 years). There are 15 patients alive and well with either unoperated or definite residual orbital disease, and their ages at presentation ranged from 27 to 78 , with most in the older age group. The presence of good vision, the patient's age, or poor general health and lack of serious symptoms were the major reasons for adopting a conservative approach in these patients.
The commonest histological type of tumour seen was a meningothelial meningioma. There were two examples each of the transitional (mixed), fibroblastic, and angioblastic types. Two patients were found at surgery to have cystic expansions of the optic nerve sheath. One had an angioblastic meningioma within the wall of the cyst, and the other had a meningioma at the orbital apex, proximal to the cystic expansion. Both these cases have been reviewed in detail elsewhere. ${ }^{2}$

Three young patients with intracranial spread were particularly interesting. We present their detailed histories.

\section{CASE 1}

This boy presented in 1972 at the age of 10 having had poor vision in the right eye from the age of 5 . He had a visual acuity of $6 / 24$ and $5 \mathrm{~mm}$ of axial proptosis, and the optic disc was atrophic. There was no family history and no cutaneous manifestations of neurofibromatosis. Ultrasonography, venography, and angiography suggested an intraconal mass. Through an anterior orbitotomy the optic nerve was exposed and seen to be diffusely enlarged in its anterior half, with an intact dura. No biopsy was taken, and a diagnosis of optic nerve glioma was made. A conservative approach was adopted and he was lost to follow-up but returned at the age of 18 with vision reduced to light perception. There were now prominent shunt vessels on the disc. A CT scan showed a heavily calcified optic nerve tumour. In addition bilateral acoustic neuromas, without clinical deafness, were found. The diagnosis was changed to optic nerve meningioma as a manifestation of the central form of neurofibromatosis. There were no cutaneous stigmata. An orbital exenteration was required to clear the orbit owing to its extensive involvement. At craniotomy a small amount of meningioma was found within the middle cranial fossa and removed in continuity with the intracanalicular optic nerve and sheath. He remains well without evidence of recurrent meningioma.

\section{CASE 2}

This young woman was first seen at the age of 20 having first noticed poor vision in the right eye at 12 years. This was attributed to amblyopia, but within four years the eye was blind. When seen by us there was $3 \mathrm{~mm}$ of proptosis and an atrophic optic disc. There was no evidence of neurofibromatosis. CT scans showed a fusiform enlargement of the optic nerve without intracranial involvement. The optic nerve was exposed via a transconjunctival approach and found to be diffusely enlarged and bluish in colour. A small biopsy was obtained. Histopathology was inconclusive without sufficient evidence for a 
diagnosis of either meningioma or glioma. However, optic nerve glioma was thought to be the more likely diagnosis, and the patient was observed. By the age of 28 a repeat CT scan demonstrated calcification of the tumour with intracranial extension. A craniotomy was performed, and a small extension of meningioma from the proximal end of the optic canal on to the jugum sphenoidale was seen. The optic nerve, its sheath, and meningioma were excised with preservation of the globe. There has been no evidence of recurrence in over two years.

\section{CASE 3}

A 20-year-old man complained of 15 months' blurred right vision. For one month he noticed that the right eye went completely blind on sustained right lateral gaze, but vision returned to normal within seconds of looking directly ahead. Central acuity was $6 / 6$, but there was only a small intact central field and defective colour vision. The disc was pale and slightly swollen, and there was $2 \mathrm{~mm}$ of axial proptosis. A CT scan showed a thickened optic nerve but no evidence of intracranial spread. Angiography showed only minor displacement of the ophthalmic artery. A craniotomy was performed, and the diagnosis of meningioma confirmed. Despite the negative radiology, tumour was found wrapped around the optic nerve for $2-3 \mathrm{~mm}$ within the middle fossa. An en bloc excision from the chiasm to the globe was achieved. There has been no evidence of recurrence over a four-year follow-up.

\section{Discussion}

Meningioma has long been recognised as a disease mainly of middle age,$^{34}$ and our series is no exception. It has also been noted that primary optic nerve sheath meningiomas appear at an earlier age than secondary orbital meningiomas or other intracranial meningiomas. ${ }^{3}$ In this series the mean age at presentation for optic nerve meningioma was $43 \cdot 2$ compared with 51.1 in 61 patients seen at the orbital clinic with sphenoid ridge meningiomas. Some or all of this age difference is presumably due to relatively smaller tumours in the orbit producing symptoms earlier than a similarly sized intracranial meningioma, especially in a region such as the sphenoid ridge.

This series also shows the usual female preponderance $(66 \%)$. The presence of significantly larger numbers of males in the younger age groups has not been recorded before, though the trend was noted in our previous paper. ${ }^{4} \mathrm{~A}$ recent paper reported a $76 \%$ incidence of progesterone receptors in a large series of meningiomas. ${ }^{5}$ This may partly explain the age and sex incidence of this tumour and the accelerated growth observed in pregnancy.
The association of neurofibromatosis with optic nerve meningioma is far less significant than for optic nerve glioma. In this series no patient had the peripheral form of the disease, and only one had the central form. In that patient the diagnosis was made after the discovery of the meningioma. Similarly, Walsh ${ }^{6}$ described three cases in children who presented with optic nerve meningiomas as the first manifestation of central neurofibromatosis, and Karp et al. ${ }^{7}$ reported on two comparable patients.

In most patients the clinical and radiological diagnosis of meningioma is readily made. Patients usually develop a central field loss with disc swelling or atrophy, sometimes with opticociliary shunt vessels; any proptosis is usually slight. The radiological features are also typical, with thickening of the optic nerve, which is often irregular in outline and is sometimes associated with calcification within the tumour surrounding the nerve.

In a minority it may be impossible to differentiate optic nerve glioma from meningioma even with axial and coronal MRI. Although carotid angiography demonstrates a tumour blush in some meningiomas, it has limited use in our experience and is not a useful means of differentiating meningiomas from gliomas. Jakobiec et al $^{8}$ using CT were able to differentiate the two tumours in $80 \%$ of patients. In recent years, using MRI in addition to CT, we have achieved a rate of differential diagnosis above $90 \%$. The correct recognition of these tumours is important in young patients, in whom gliomas are relatively common, and meningiomas, though rarer, behave in a more aggressive and invasive manner and should be removed as early as possible. Biopsy should prove which lesion is present, but the differentiation of the two can be difficult unless substantial amounts of tissue are obtained. Often the presence of fairly good vision prevents this from being done. A possible solution to this dilemma is discussed in the adjoining paper on optic nerve glioma.

The different behaviour of optic nerve meningiomas in the young compared to the middle aged and elderly in our series is an important observation in relation to their management. Walsh ${ }^{6}$ and others ${ }^{79}$ have previously observed that optic nerve meningiomas in the young can behave in an aggressive manner, and our results support this view. Our findings that four of six patients under 20 years of age and nine of 20 patients in the next two decades developed involvement of the middle cranial fossa is particularly disturbing when compared with the behaviour of the tumour in subsequent decades (four of 24 patients over 40 developed intracranial disease). This difference is, we presume, due to a more active level of biological activity of the meningothelial cells, though we have no evidence of a different histopath- 
ological picture in the two groups. The difference is not produced by longer observation of the younger patients, for our older patients have been watched for as long (range 1-15 years in both groups, median 8 years in the older group and 4.5 years in the younger). Involvement of the optic canal and the posterior surface of the sphenoid occurred in 17 of our patients. Karp et al ${ }^{7}$ recorded eight of 25 patients presenting under 20 years of age, three at least of whom had intracranial extension. Spread to the middle cranial fossa must have a grave effect on prognosis, for it is almost impossible to eradicate meningioma once the sphenoid is involved. Surgical removal or radiotherapy may slow the spread of the tumour, but it invariably recurs. Only lengthy periods of observation of these patients will provide evidence of the nature and rate of this spread and the effect of attempts at treating these tumours.

This experience has altered our method of dealing with these tumours. Previously surgery was directed at either decompressing the optic nerve to preserve vision, or excision by stripping the nerve of meningioma, or removing the affected part of the optic nerve together with the surrounding tumour. Incising dura to release pressure on the nerve failed to slow visual deterioration and spread the meningioma into the orbital fat and extraocular muscles, so that exenteration was then the only method of removing the tumour from the orbit. We have not used this technique since $1979 .{ }^{4}$ Stripping meningioma off the nerve has always resulted in total loss of remaining vision, though others have reported preservation of vision in some patients. "11 Frequently we have excised the optic nerve together with the tumour. Unfortunately three of 10 such patients have subsequently suffered sphenoidal involvement, two of them being under 40 at presentation.

Our current management is influenced by the behaviour of the tumour in patients of differing age. Patients whose symptoms begin when they are over 40 years have a lesion which should progress slowly, and there is only a small risk of spread to the middle fossa. Many of these patients do not need treatment, but if the tumour enlarges and produces disfiguring proptosis then either radiotherapy or local excision will improve cosmesis and reduce the chances of recurrence within the orbit. Smith et al, ${ }^{12}$ and more recently Kennerdell et al, ${ }^{13}$ have reported on the efficacy of radiotherapy in slowing the progression of optic nerve meningioma, and we have used their technique on two patients with initially encouraging results.

Patients under 40 years should, we believe, be treated more vigorously. All, eventually, should have the optic nerve excised. If the patient is younger than 30 then the chances of intracranial spread are considerable. We believe that the tumour should be totally excised at an early stage by a transfrontal craniotomy, so that the whole of the optic nerve from the chiasm to the globe can be removed with its sheath even when vision is relatively good. Between the ages of 30 and 40 years the threat of extension to the sphenoid is somewhat less; these patients can be watched until vision is severely impaired before neurosurgically excising the optic nerve and tumour. In all patients, if there is any doubt on clinical or neuroradiological grounds that the lesion is not a meningioma, then histopathological proof must be obtained, usually by frozen section, before embarking on extensive excision. If the tumour has involved the optic canal and the dorsum sphenoidale, surgical debridement can be pursued and the patient subsequently treated with radiotherapy.

Our study supports the view that the characteristics of primary optic nerve meningioma vary with the age of the patient. In general the younger the patient the more active the meningioma. Therapy should therefore recognise this variation in biological activity, with infrequent intervention in the middle aged and elderly and early excision in the young.

Our thanks are due to all the clinicians who referred these patients, Mr Alan Crockard and Professor Lindsay Symon, neurosurgeons at the National Hospitals, Queen Square and Maida Vale, Dr G A S Lloyd and Dr Ivan Moseley, Radiology Department, the staff of the Medical Illustration Department, and Mrs S J Cole who helped with the typing of the manuscript

AAMcN was supported by the Royal Australian College of Ophthalmologists-OPSM Travelling Fellowship.

\section{References}

1 Bradbury P, Levy IS, McDonald WI. Transient uniocular visual loss on deviation of the eye in association with intraorbital tumours. J Neurol Neurosurg Psychiatry 1987; 50: 615-9.

$2 \mathrm{McNab}$ AA, Wright JE. Cysts of the optic nerve. Three cases associated with meningioma. Eye in press.

3 Spencer WH. Primary neoplasms of the optic nerve and its sheaths: clinical features and current concepts of pathogenetic mechanisms. Trans AM Ophthalmol Soc 1972; 70: 490-528.

4 Wright JE, Call NB, Liaricos S. Primary optic nerve meningioma. Br J Ophthalmol 1980; 64: 553-8.

5 Lesch KP, Fahlbusch R. Simultaneous estradiol and progesterone receptor analysis in meningiomas. Surg Neurol 1986; 26: 257-63.

6 Walsh FB. Meningiomas primary within the orbit and optic canal. In: Smith JL, ed. Neuro-ophthalmology symposium of the University of Miami and the Bascom Palmer Eye Institute. St Louis: Mosby, 1970; 5: 240-66.

7 Karp LA, Zimmerman LE, Borit A, Spencer W. Primary intraorbital meningiomas. Arch Ophthalmol 1974; 91: 24-8.

8 Jakobiec FA, Depot MJ, Kennerdell JS, et al. Combined clinical and computerised tomographic diagnosis of orbital glioma and meningioma. Ophthalmology 1984; 91: 137-55.

9 Alper MG. Management of primary optic nerve meningiomas: current status - therapy in controversy. J Clin Neuro Ophthalmol 1981: 1: 101-17.

10 Mark KE, Kennerdell JS, Maroon JC, et al. Microsurgical removal of a primary intraorbital meningioma. Am J Ophthalmol 1978; 37: 704-9. 
11 Ebers GC, Girvin JP, Canny CB. A 'possible' optic nerve meningioma. Arch Neurol 1980; 37: 781-3.

12 Smith JL, Vuksanovic MM, Yates BM, Bienfang DC. Radiation therapy for primary optic nerve meningiomas. J Clin Neuro Ophthalmol 1981: 1: 85-9.
13 Kennerdell JS, Maroon JC, Malton M, Warren FA. The management of optic nerve meningiomas. Am J Ophthalmol 1988; 106: 450-7.

Accepted for publication 25 May 1989. 